\title{
Phenotypic Characterization of Enzymatic and Non- enzymatic Virulence Factors in Etiopathogenesis and Evolution of Drug Resistance among Dermatophytes
}

\author{
Shanmuga Priya $C^{1}\left(\mathbb{D}\right.$, R. Prabha ${ }^{1 *}$ (D) and M. Rajesh ${ }^{2}$ (iD \\ ${ }^{1}$ Department of Microbiology, Mahatma Gandhi Medical College and Research Institute,Sri Balaji Vidyapeeth, \\ (Deemed to be university), Pillaiyarkuppam, Puducherry - 607 402, India. \\ ${ }^{2}$ Department of Dermatology, Venerology and Leprosy,Mahatma Gandhi Medical College and Research \\ Institute,Sri Balaji Vidyapeeth, (Deemed to be university), Pillaiyarkuppam, Puducherry - 607 402, India.
}

\begin{abstract}
The virulence factors of dermatophytes play a vital role in the etio-pathogenesis and emergence of drug resistance. Regular surveillance is essential for constant monitoring of association of virulence factors in the emergence of drug resistant isolates and to guide proper therapeutic strategies to combat infections due to drug resistant isolates of dermatophytes. The aim of this study is to isolate and identify various enzymatic and non-enzymatic virulence factors of dermatophytes isolated from clinically suspected cases of dermatophytosis attending the dermatology outpatient clinic. From 30 clinical samples received from department of Dermatology to the department of Microbiology during the study period July to December 2019, $\mathrm{KOH}$ mount and fungal culture was done and only the isolates which grew dermatophytes were used for detecting virulence factors using standard methods. Among 30 affected patients, 13 were male (43.3\%) and 17 were female (56.7\%) with age range of 13 to 60 years. Skin sample yielded positive growth in $96.6 \%$ of the cases. Out of 30 samples, 6 samples were positive for Trichophyton mentagrophytes. Tinea corporis was the common manifestation which yielded positive growth. Female predominance was seen. Male predominance was seen in younger age group while females were more commonly affected in older age group. Adolescents were commonly affected age group (40\%). Then people between age 41-50 (middle age group) were mostly affected. The six Trichophyton mentagrophytes were found to have enzymatic and non- enzymatic virulence factors. All the virulence factors included in the study were present in Trichophyton mentagrophytes.

Keywords: Anti-fungal susceptibility, Trichophyton mentagrophytes, Tinea corporis
\end{abstract}

*Correspondence: philomeena.rajendran@gmail.com; +91 9629769060

(Received: June 29, 2020; accepted: October 21, 2020)

Citation: Shanmuga PC, Prabha R, Rajesh M. Phenotypic Characterization of Enzymatic and Non-enzymatic Virulence Factors in Etiopathogenesis and Evolution of Drug Resistance among Dermatophytes. J Pure Appl Microbiol. 2020;14(4):2661-2667. doi: 10.22207/JPAM.14.4.43

(C) The Author(s) 2020. Open Access. This article is distributed under the terms of the Creative Commons Attribution 4.0 International License which permits unrestricted use, sharing, distribution, and reproduction in any medium, provided you give appropriate credit to the original author(s) and the source, provide a link to the Creative Commons license, and indicate if changes were made. 


\section{INTRODUCTION}

Dermatophytes are known as fungal agents causing infections in immunocompromised individuals and also nosocomial infections. Based on the habitat, dermatophytes are divided into three groups like: Zoophilic (animal), anthropophilic (humans) and geophilic (soil). In developing countries like India, dermatophytic infections are predominantly seen. In addition the disease is more common in this country because of the prevalence of hot and humid weather ${ }^{1}$. Over the past few years, the prevalence and incidence of dermatophytosis is increasing at an increasing rate across the country ${ }^{2}$. The immunity of the host will determine the disease outcome which ranges from cutaneous or subcutaneous to invasive, disseminated, and life-threatening infections ${ }^{3}$.

Clinical cure is associated with development of cell mediated immunity. Pathogenesis of dermatophytes involves mainly three steps like adherence to the host tissue, invasion, and progression of host immune response. Pathogenesis is also due to production of virulence factors which may be involved in the emergence of anti-fungal resistance. Studies have been done on the production of virulence factors like protease, lipase enzyme and hemolytic (CAMPlike) activities of the dermatophytes.

Proteolytic effects have been identified and also differentiated from bacterial proteolytic effects. The enzyme is helpful to characterize and identify the microbial proteases and play a major role in pathogenesis of infectious diseases. Extracellular enzymes like keratinase, elastase, protease, phospholipase, lipase, hemolysins and CAMP like factors are produced by dermatophytes using plate assay technique. Hemolytic factor plays a vital role in dermatophytic infections because of the cytotoxic effects on RBC as well as phagocytic cells which results in the formation of pore and lysis ${ }^{4}$. Important fungal pathogens like Trichophyton mentagrophytes, Trichophyton rubrum, Microsporum canis and Microsporum gypseum were more commonly isolated from the keratin substances in hair with production of keratinase activity.

In the past few years, antifungal resistance has emerged because of the irrational use of antifungal agents in cutaneous mycotic infections $s^{5}$. Routine monitoring is essential for the surveillance of drug resistant isolates and also for timely tracking of virulence factors on dermatophytic fungal infections to pay a way for proper treatment. So to know the exact picture of dermatophytosis in India, there is need for more studies in vitro ${ }^{6}$. The present study was to phenotypically characterize the enzymatic and non-enzymatic virulence factors, and evolution of drug resistance among clinical isolates of dermatophytes.

\section{MATERIALS AND METHODS Samples}

A total of 200 patients with dermatophytosis visited OPD of Department of Dermatology at Mahatma Gandhi Medical College \& Research Institute, Pondicherry, India during July to December 2019. Clinical samples viz skin, nail, and tissues of clinically suspected patients with dermatophytosis were collected from the collaborative department of Dermatology and sent to mycology laboratory separately for microscopic examination and culture. These samples were also sent to Department of Microbiology for culturing the clinical specimens collected from patients with dermatophytosis. This analytical study was carried out in the department of Microbiology.

These 30 samples were parallely observed with microscope using Potassium hydroxide $(\mathrm{KOH})$ mount and then it was inoculated simultaneously in plain Sabourauds Dextrose agar and Sabourauds Dextrose agar with chloramphenicol $(30 \mu \mathrm{g})$, Gentamicin $(30 \mu \mathrm{g})$, and Cycloheximide in duplicate. These inoculated plates were incubated at $25^{\circ} \mathrm{C}$ and $37^{\circ} \mathrm{C}$ and were regularly monitored for about six weeks. The growth of the dermatophytes were identified based on their colony morphology, microscopic examination by using lacto phenol cotton blue (LPCB) mount. Among 200 patients visited OPD of Dermatology department, 30 patients clinically diagnosed for dermatophytosis irrespective of their age and sex and who were not undergoing treatment for the same were included in the study.

\section{Enzymatic virulence factors}

The phenotypic identification of enzymatic virulence factors were performed for these dermatophyte isolates according to the following methods: Phospholipase and Protease production was done using Candida albicans 
as positive control. The dermatophytes were inoculated on egg yolk agar and incubated at $25^{\circ} \mathrm{C}$ for 2 weeks, clear halo zone of precipitation around the colony indicates phospholipase enzyme production? ${ }^{7}$ For Gelatinase (protease) production, the dermatophytes were stab inoculated into gelatin tubes and incubated at $25^{\circ} \mathrm{C}$ for 2 weeks. Formation of clear zones around the colonies indicates gelatinase enzyme production. Staphylococcus aureus was used as positive control'.

For Lipase enzyme Malassezia furfur was used as positive control. The fungal isolates were inoculated on lipase medium and incubated at $25^{\circ} \mathrm{C}$ for 2 weeks and these tests appeared as a clear halo zone of precipitation around the colony ${ }^{7}$. Keratin powder was added to trypticase Soy Agar (TSA) and adjusted to $\mathrm{pH}$ 5.6. Fungal isolates were cultured on TSA medium and incubated at $25^{\circ} \mathrm{C}$ for 2 weeks. Keratinase activity was observed by incubating the plates at $25^{\circ} \mathrm{C}$ for 7 days and were looked for a clear zone around the colonies ${ }^{8}$. Elastase production was carried out with saturated ammonium sulfate solution which was added to the fungal isolates. 1 g elastin powder was added to Trypticase Soy Agar. The dermatophytes were inoculated on elastase test medium and incubated at $25^{\circ} \mathrm{C}$ for 2 weeks. Appearance of clear zone around the growth indicates enzyme production?.

\section{Non-enzymatic virulence factors}

The phenotypic identification of nonenzymatic virulence factors like hemolysin was detected using Colombia agar with $5 \%$ sheep blood and the plate was incubated at $37^{\circ} \mathrm{C}$ for $1-5$ days as explained by Schaufuss et al. ${ }^{10}$ Production of CAMP like factor (Co-hemolytic activity) was performed using the standard strains of bacteria S.aureus (ATCC 25923). After incubation time for hemolytic activity, the edge of a loop was used to streak each bacterium in straight lines across the plate at a distance of $10 \mathrm{~mm}$ from the border of dermatophyte colony. The plates were then incubated at $25^{\circ} \mathrm{C}$ and inspected daily for 1-7 days. A distinct arrow head zone of hemolysis at the intersection of the standard strain and the test dermatophte colony was considered as positive result for CAMP-like reactions ${ }^{10}$.

Statistical Analysis

Mean and standard deviation were calculated for numerical variables. Continuous variables were compared using Student's $t$-test for normally distributed variables.

\section{RESULTS AND DISCUSSION}

Dermatophytosis is one of the major causes of fungal infection in India among the patients with diabetes and other diseases. Transmission of dermatophytes from man to man indirectly via fomites, overcrowded human setting in developing countries, rearing and close proximity to domestic pets are the main risk factors for the transmission of dermatophytes in developed countries.

The enzymatic and non-enzymatic virulence factors of the organisms play a major role in these infections. Among 200 patients visited during the six months from July to December 2019 to OPD, Department of Dermatology, 30 were suspected with dermatophytic infections. Among 30 patients, 13 were male (43.3\%) and 17 were female $(56.7 \%)$ presented with age range from 13to 60years (Fig: 1), 8 males and 4 females were infected in the age group 11-20 yrs, 3 males and 2 females in the age group 21-30 yrs, 1 male and 4 females in the age group 31-40 yrs, 1 male and 6 females in 41-50 yrs age group and one female in $50-60$ yrs age group. About $40 \%$ of the adults were more commonly infected, 2 samples were positive in the age group 11-20 yrs, 1 sample in the age group 21 to $30 \mathrm{yrs}, 1$ sample in the age group 31-40 yrs and 1 sample in the age group 41 to $50 \mathrm{yrs}$. This is in accordance to the other study where maximum number of cases $(75 \%)$ encountered was in the age group of 11-20 yrs and 21-30 years ${ }^{11}$. In another study done at Karnataka, majority of affected people belonged to the age group 21-30 yrs (10 cases of Tinea corporis) $)^{12}$. As regards to the age, maximum number of cases was in the age group of 16-30 years in another study ${ }^{13}$. In the present study, infection was more prevalent among females compared to males (Fig: 2). This could be due to the fact that females are commonly involved in occupations like, gardening, taking care of domestic livestock etc. But in a study by Sentamil selvi, the higher incidence was found in males which could be due to greater physical activity and increased sweating ${ }^{14}$. In another study also more cases were reported among males ${ }^{15}$. Our study is similar to the study done by Gebreabiezgi 
et al where it has been reported that more number of females are affected than males ${ }^{16}$.

In this study many clinical specimens like hair, skin, nail and tissues were received in the mycology laboratory. Skin was the predominant clinical manifestation in $96.6 \%$ of the cases (Fig: 3). This is similar to the study where out of 150 children recruited in the study and examined for Tinea infections, $81.3 \%$ (122 persons) were found to have skin lesions caused by dermatophytes ${ }^{17}$. All six samples were taken from patients with Tinea corporis in our study. Nearly, $66.6 \%$ of Tinea corporis was identified and $10 \%$ of patients presented with the dermatophytic infections (Fig: 4) which is similar to the study done by Surendar et al where they have reported Tinea corporis as the commonest clinical manifestation $(44.3 \%)^{13}$. In the studies by Veena timmappa et al, Tinea corporis was reported as the commonest presentation $(40.5 \%)^{18}$. But Tinea cruris was the most common clinical presentation (23.4\%) followed by Tinea corporis $14 \%$ in a study done by Ujjwal kumar et $\mathrm{al}^{19}$.

Tinea pedis (29.2\%) was reported as common dermatophytosis followed by Tinea cruris (26.2\%) in another study on superficial mycosis

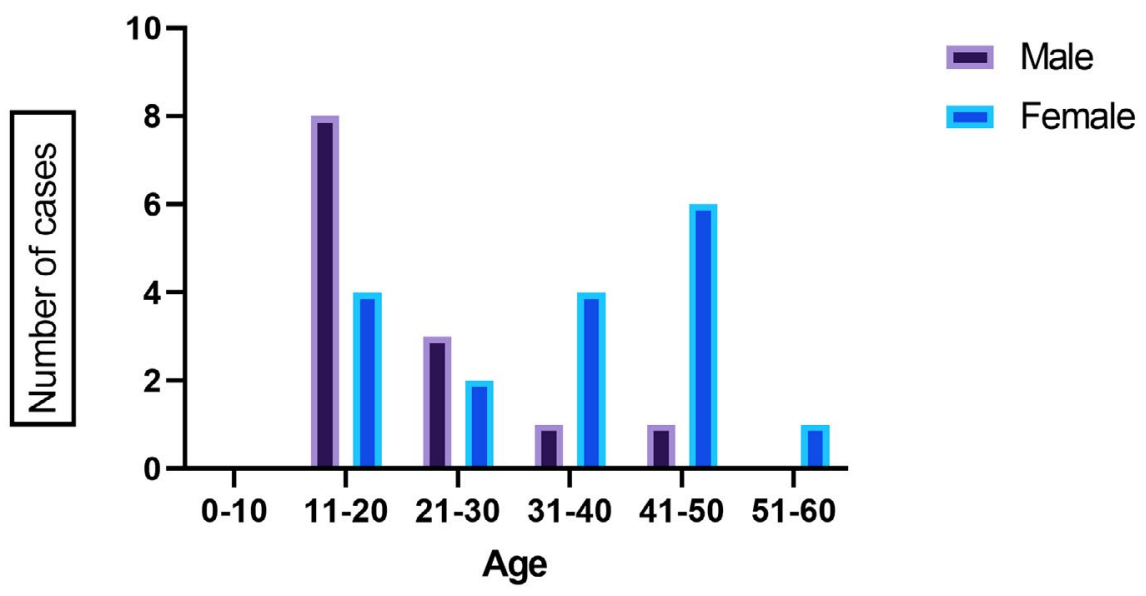

Fig. 1. Distribution of patients according to sex

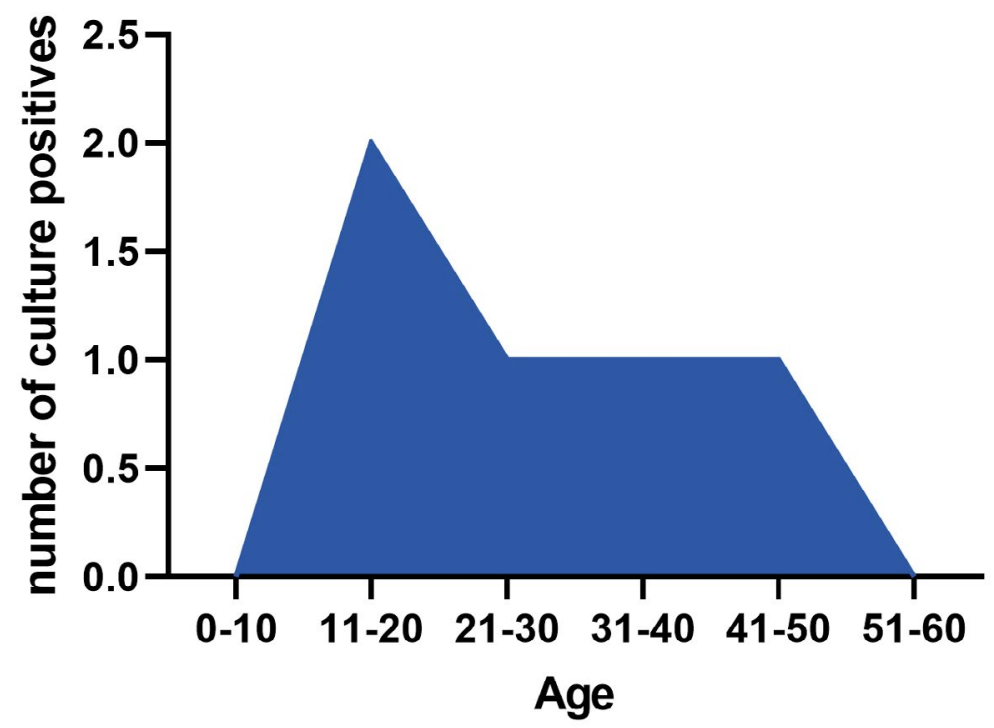

Fig. 2. Distribution Of Patients According To Age 
in a hospital in north-east India, which differs from our study ${ }^{20}$. Most common clinical type was Tinea corporis 66(.5\%) followed by Tinea cruris 25(19.23\%), Tinea unguium 18(13.84\%) and Tinea capitis $10(07.69 \%)$ in a study done by Manjunath et $\mathrm{al}^{21}$.

All the three genera of dermatophytes, that is, Trichophyton, Epidermophyton, and Microsporum were isolated as causative agents of infection in a study conducted by Surendran et $\mathrm{al}^{13}$. All the six samples were positive for Trichophyton mentagrophytes (Fig: 3). In a study done by Imran majid et al, Trichophyton rubrum $55 \%$ and Trichophyton tonsurans 20\% were reported as common dermatophytes ${ }^{22}$. Trichophyton verrucosum was reported as commonest species (26 cases), followed by Trichophyton rubrum (15 patients), and Trichophyton mentagrophytes (8 cases) in a study done by Sudip das ${ }^{23}$. Another study reported Trichophyton mentagrophytes as the predominant dermatophytes infecting the humans $13(32.5 \%)^{24}$. In another study, of the 130 dermatophytes isolates $72.3 \%$ was accounted by Trichophyton violaceum, Trichophyton mentagrophytes and Trichophyton tonsurans ${ }^{16}$. Among the three dominant species, Trichophyton violaceum accounted for $37.69 \%$ of the total isolates in a study done in Ethiopia ${ }^{25,26}$

All six clinical isolates were tested for the enzymatic production of phospholipase, lipase, protease, keratinase, gelatinase and nonenzymatic factors like hemolysin and CAMP-like factors which showed strong enzymatic and nonenzymatic activities (Fig: 5). Our study is similar to

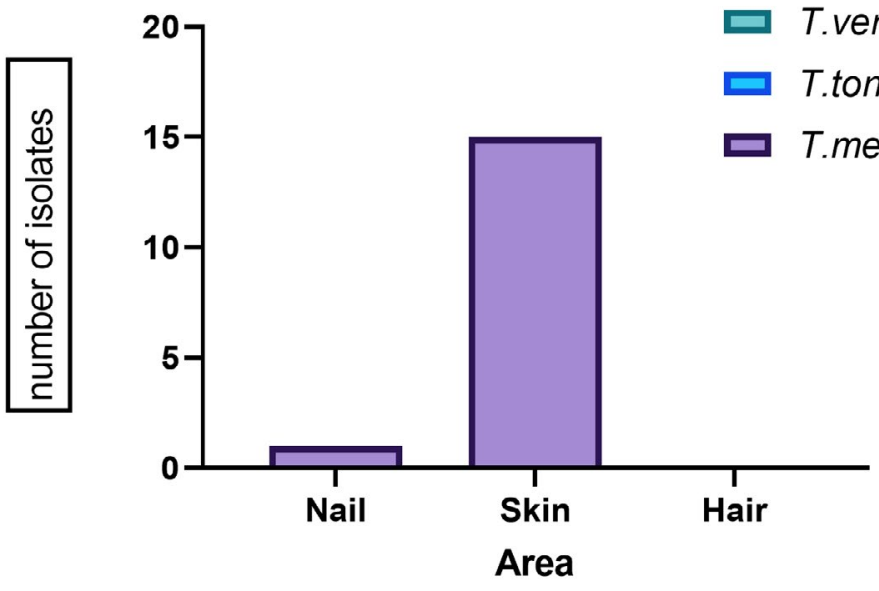

Fig. 3. Distribution of samples

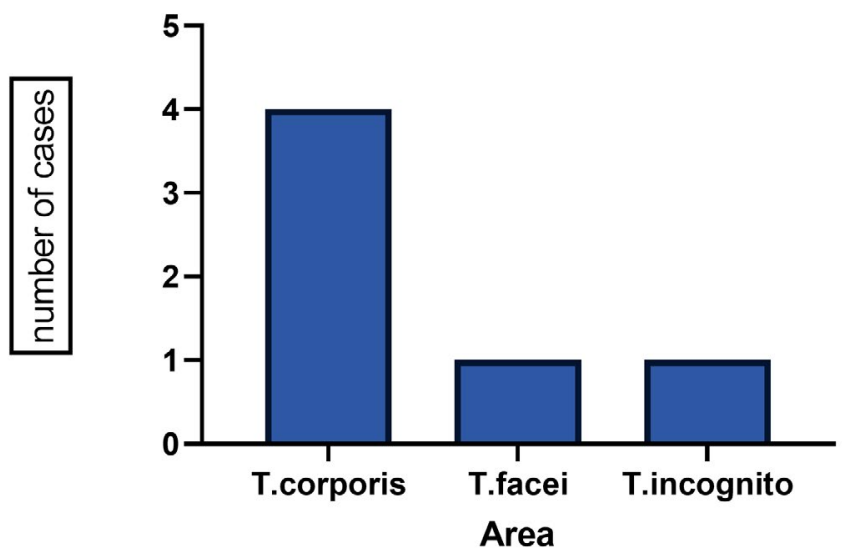

Fig. 4. Clinical type of dermatophytoses in present study 


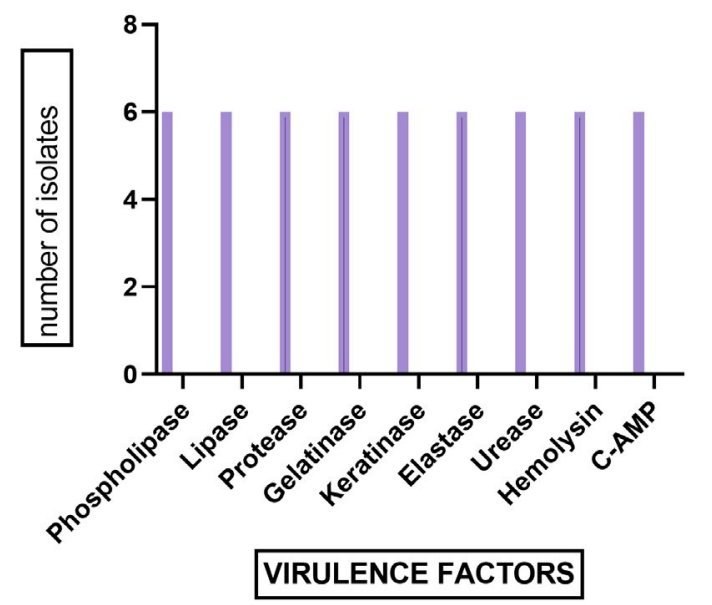

Fig. 5. Distribution of various virulence factors

another study where all the isolates had CAMPpositive reaction with S.aureus. All the isolates expressed proteolytic activity ${ }^{27}$.

An enzyme called lipase, plays a major role in the early stages of infection initially by breaking the lipid surface layer and colonizing the stratum corneum of the skin .The enzymatic virulence factors like phospholipase, lipase, proteinase, gelatinase, elastase and the nonenzymatic factors like hemolysin and CAMP-like factor production plays vital role as biomarker for identification of dermatophytes. Our study is in par with study done by Elavarasi et al where the virulence activity of phospholipase, lipase, protease and gelatinase was observed in all the dermatophyte species ${ }^{7}$.

\section{CONCLUSION}

Dermatophyte infections are very common in our country due to hot, humid climate and poor hygienic conditions.

The enzymatic and non-enzymatic virulence factors of dermatophytes contribute a major role in emergence of anti-fungal drug resistance. Our study emphasizes the importance of identifying these virulence factors among clinical isolates of dermatophytes. The emergence of resistance may reflect poor antifungal stewardship. Periodic monitoring of fungal profile is essential. Strict policy should be formulated to avoid irrational use of anti-fungal agents responsible for emergence of drug resistance.

\section{ACKNOWLEDGMENTS}

I sincerely thank Honorable Chancellor, Vice-Chancellor, Dean-Research, Sri Balaji Vidyapeeth (Deemed-to-be-University) and Dean of Mahatma Gandhi Medical College and Research Institute, for providing the facilities for this ShortTerm Student ICMR Research Project (Reference ID: 2019-01257).

\section{CONFLICT OF INTEREST}

The authors declare that there is no conflict of interest.

\section{AUTHORS' CONTRIBUTION}

Research conception \& design: SP, PR, RM, Performing the experiments: $S P$, Data acquisition: SP, PR, RM, Data analysis and interpretation: $S P, P R$, RM, Drafting of the manuscript: $P R, R M$, Critical revision of the manuscript: $S P, P R, R M$, Receiving grant: SP Approval of final manuscript: all authors.

\section{FUNDING}

Received funding for student short term project from Indian Council of Medical Research (ICMR).

\section{DATA AVAILABILITY}

Data available in the Department of Microbiology, Mahatma Gandhi Medical College \& Research Institute, Pondicherry. Could be provided if necessary, to anyone. 


\section{ETHICS STATEMENT}

This research work has been approved by our Institutional Human Ethics Committee (IHEC).

\section{REFERENCES}

1. Bhatia VK, Sharma PC. Epidemiological studies on Dermatophytosis in human patients in Himachal Pradesh, India. Springer Plus. 2014;3:134. doi: 10.1186/2193-1801-3-134

2. Verma S, Madhu R. The Great Indian Epidemic of Superficial Dermatophytosis: An Appraisal. Indian J Dermatol. 2017;62(3):227-36.

3. Kohler JR, Hube B, Puccia R, Casadevall A, Perfect JR. Fungi that infect humans. Microbiol Spectr. 2016;5(3):10.

4. Gnat S, Lagowski D, Nowakiewicz A, Zieba P. Phenotypic characterization of enzymatic activity of clinical dermatophyte isolates from animals with and without skin lesions and humans. J Appl Microbiol. 2018;125(3):700-709. doi: 10.1111/jam.13921

5. Martinez-Rossi NM, Bitencourt TA, Peres NTA, et al. Dermatophyte Resistance to Antifungal Drugs: Mechanisms and Prospectus. Front Microbiol. 2018; 29;9:1108. doi: 10.3389/fmicb.2018.01108

6. Dogra S, Uprety S. The menace of chronic and recurrent dermatophytosis in India: Is the problem deeper than we perceive? Indian Dermatol Online J. 2016;7:73-76. doi: 10.4103/2229-5178.178100

7. Elavarashi E, Kindo AJ, Rangarajan S. Enzymatic and NonEnzymatic Virulence Activities of Dermatophytes on Solid Media. J Clin Diagn Res JCDR. 2017;11(2):DC23-5. doi: 10.7860/JCDR/2017/23147.9410

8. Afshari MA, Shams-Ghahfarokhi M, Razzaghi-Abyaneh M. Antifungal susceptibility and virulence factors of clinically isolated dermatophytes in Tehran, Iran. Iran J Microbiol. 2016;8(1):36-46.

9. Muhsin TM, Aubaid AH, Al duboon A H. Extracellular enzyme activities of dermatophytes and yeast isolates on solid media. Mycoses. 1997;40(11-12):465-469.doi: 10.1111/j.1439-0507.1997.tb00186.x

10. Schaufuss P, Brasch J, Steller U. Dermatophytes can trigger cooperative (CAMP like) haemolytic reactions. Br J Dermatol. 2005;153(3): 584-590. doi: 10.1111/j.1365-2133.2005.06679.x

11. Kakande $T$, Batunge $Y$, Eilu E, et al. Prevalence of Dermatophytosis and Antifungal Activity of Ethanolic Crude Leaf Extract of Tetradenia riparia against Dermatophytes Isolated from Patients Attending Kampala International University Teaching Hospital, Uganda. Dermatology Research and Practice. 2019;2019:1-13. doi: 10.1155/2019/9328621

12. Noronha TM, Tophakhane RS, Nadiger S. Clinicomicrobiological study of dermatophytosis in a tertiarycare hospital in North Karnataka. Indian Dermatol Online J. 20161;7(4):264-271. doi: 10.4103/22295178.185488

13. Surendran K, Bhat RM, Boloor R, Nandakishore B, Sukumar D. A Clinical and Mycological Study of Dermatophytic Infections. Indian J Dermatol.
2014;59(3):262-267. doi: 10.4103/0019-5154.131391

14. Sentamilselvi G, Kamalam A, Ajithadas K, Janaki C, Thambiah AS. Scenario of chronic dermatophytosis: an Indian study. Mycopathologia. 1997-1998;140(3):129135. doi: 10.1023/A:1006843418759

15. Bhatia VK, Sharma PC. Epidemiological studies on Dermatophytosis in human patients in Himachal Pradesh, India. Springer Plus. 2014;3:134. doi: 10.1186/2193-1801-3-134

16. Teklebirhan G, Bitew A. Prevalence of Dermatophytic Infection and the Spectrum of Dermatophytes in Patients Attending a Tertiary Hospital in Addis Ababa, Ethiopia. Int J Microbiol. 2015;1-5:653419. doi: 10.1155/2015/653419

17. Jejidah, John muthini, Anthony. Prevalence of Tinea capitis in school going children in Mathare, informal statement in Nairobi, Kenya. BMC Res Notes. 2015;8:274.

18. Thimmappa V, Shastry V, Betkerur J. Clinicomycological study and comparison of efficacy of three different techniques of sample collection from skin lesions for potassium hydroxide mount preparation in dermatophytoses. Int J Res Dermatol. 2019;5(1):88-93. doi: 10.18203/issn.2455-4529.

19. Kumar U, Chauhan MPS, Varma K. "A clinico epidemiological study of dermatophytosis in a tertiary care center, Ujjain." IP Indian J Clin Exp Dermatol. 2019;5(1):89-92. doi: 10.18231/2581-4729.2019.0019

20. Grover S, Roy P. Clinico-mycological Profile of Superficial Mycosis in a Hospital in North-East India. Med J Armed Forces India. 2003;59(2):114-116. doi: 10.1016/S0377-1237(03)80053-9

21. Manjunath M, Mallikarjun K, Dadapeer. Clinicomycological study of dermatomycosis in a tertiary care hospital. Indian J Microbiol Res. 2016;3(2):190193. doi: 10.5958/2394-5478.2016.00043.1

22. Majid I, Sheikh G, Kanth F, Hakak R. Relapse after Oral Terbinafine Therapy in Dermatophytosis: A Clinical and Mycological Study. Indian J Dermatol. 2016;61(5):529533. doi: 10.4103/0019-5154.190120

23. Das S, De A, Saha R, et al. The current Indian epidemic of dermatophytosis: A study on causative agents and sensitivity patterns. Indian J Dermatol. 2020;65(2):118122. doi: 10.4103/ijd.IJD_203_19

24. Pakshir K, Leila B, Rezaei Z, Manuchehr S, Zomorodian K. In vitro activity of six antifungal drugs against clinically important dermatophytes. Jundishapur J Microbiol. 2009;2(4):158-163.

25. Tinea capitis in south-western Ethiopia: a study of risk factors for infection and carriage. Int $J$ Dermatol. 1997;36(9):661-666. doi: 10.1046/j.13654362.1997.00236.x

26. Woldeamanuel $Y$, Mengistu Y, Chryssanthou E, Petrini B. Dermatophytosis in Tulugudu Island, Ethiopia. Med Mycol. 2005;43(1):79-82. doi: 10.1080/13693780410001711981

27. Pakshir K, Mohamadi T, Khodadadi H, et al. Proteolytic activity and cooperative hemolytic effect of dermatophytes with different species of bacteria. Curr Med Mycol. 2016;2(4):9-14. 